Bundesgesundheitsbl 2009 · 52:1037-1044 DOI 10.1007/s00103-009-0957-7

Online publiziert: 10. September 2009

(c) Springer-Verlag 2009

\author{
S. Reiter $\cdot$ C. Poethko-Müller \\ Abteilung für Infektionsepidemiologie, Fachgebiet \\ Impfprävention, Robert Koch-Institut, Berlin
}

\title{
Aktuelle Entwicklung von Impfquoten und Impflücken bei Kindern und Jugendlichen in Deutschland
}

Impfungen sind effektive und sichere Maßnahmen der Primärprävention und tragen maßgeblich zum Infektionsschutz und damit zur Verbesserung der Gesundheit der Bevölkerung bei. Ein optimaler individueller und kollektiver Impfschutz kann aber nur dann erreicht werden, wenn frühzeitig hohe Impfquoten erzielt werden.

Die Ständige Impfkommission (STI$\mathrm{KO}$ ) empfiehlt in den ersten beiden Lebensjahren für alle Kinder eine Grundimmunisierung gegen insgesamt zwölf Erreger: Diphtherie, Tetanus, Pertussis, Haemophilus influenza Typ $b$ (Hib), Poliomyelitis, Hepatitis B, Pneumokokken, Masern, Mumps, Röteln, Varizellen und Meningokokken C. Ein früher Impfschutz ist vor allem für Hib und Pertussis notwendig, da bei diesen Erkrankungen die höchste Morbidität und Mortalität Kinder im ersten Lebensjahr betrifft. Ebenso sind zeitgerechte Impfungen gegen Masern, Mumps, Röteln (MMR) sowie Varizellen empfohlen, da für diese Krankheiten der Nestschutz schon früh im ersten Lebensjahr nachlässt und Komplikationen bei Varizellen-Erkrankungen häufig auch kleine Kinder betreffen.

Im Alter von fünf bis sechs Jahren wird eine erste Auffrischimpfung gegen Diphtherie, Tetanus und Pertussis empfohlen. Alle Jugendlichen sollen im Alter von neun bis 17 Jahren weitere vier Auffrischimpfungen erhalten: die zweite Tetanus-, zweite Diphtherie-, zweite Pertussisund die erste Polio-Auffrischimpfung. Für Mädchen wird seit 2007 eine dreimalige Impfung gegen humane Papillomaviren (HPV) empfohlen. Fehlende Impfungen sollen - mit Ausnahme der Impfungen gegen Pneumokokken und Hib - bis zum 18. Lebensjahr nachgeholt werden [1].

Die konsequente und zeitgerechte Umsetzung des STIKO-Impfplans stellt eine Herausforderung für alle beteiligten Akteure der Impfprävention dar. Die kontinuierliche Dokumentation und Analyse des Impf- und Immunstatus und Kenntnisse über die Einstellungen und die Inanspruchnahme von Impfungen in der Bevölkerung sind eine wichtige Voraussetzung, um die Umsetzung der STIKOEmpfehlungen beurteilen und Impflücken erkennen zu können.

Ziel des vorliegenden Beitrages ist es, anhand der verfügbaren Datenquellen aktuelle Impfquoten und Impflücken bei Kindern und Jugendlichen in Deutschland aufzuzeigen. Im Gegensatz zu anderen europäischen Ländern existiert in Deutschland kein Impfregister, das eine zeitnahe, kontinuierliche und vollständige Erfassung des Impfstatus der Bevölkerung ermöglicht [2]. Zusätzliche Analysen der Daten aus dem Kinder- und Jugendgesundheitssurvey (KiGGS) beschreiben den Impfstatus zum Ende des zweiten Lebensjahres, die Zeitgerechtigkeit von Pertussis-Impfungen im Säuglingsalter sowie Defizite bei den Pertussis- und Polioauffrischungsimpfungen.

\section{Impfschutz bei Aufnahme in den Kindergarten}

In einigen Bundesländern wird beim Eintritt von Kindern in eine Kindertagesstätte (KiTa) eine ärztliche Bescheinigung verlangt, die auch Auskunft über ihren aktuellen Impfschutz gibt. Da mit dem Kindergartenbesuch das Infektionsrisiko steigt, stellt dieser frühe Zeitpunkt eine gute Gelegenheit zur Impfstatusüberprüfung und Impflückenschließung dar. Die Analyse der Daten aus SchleswigHolstein von insgesamt 18.468 Kindern im Alter von ein bis fünf Jahren zeigt für das Jahr 2007 sehr hohe Impfquoten gegen Diphtherie (96,9\%), Tetanus (97,7\%) und Polio $(96,4 \%)$. Waren im Jahr 2000 nur $75 \%$ gegen Hepatitis B geimpft, so waren es 2007 bereits 90,9\%. Auch der 2007 erreichte Impfschutz bei Hib (94,9\%) und Pertussis (93,7\%) konnte gegenüber den Vorjahren deutlich verbessert werden. Der Anteil der Kindergartenkinder mit einer zweiten Masernimpfung hat sich von 14,1\% im Jahr 2000 auf 82,3\% im Jahr 2007 erhöht. Gegen Varizellen waren in einer kleineren Teilstichprobe 57,9\% einmal geimpft. In den Kreisen in SchleswigHolstein wurden allerdings große regionale Unterschiede bei den erzielten Impfquoten festgestellt [3].

\section{Impfquoten bei den Schuleingangsuntersuchungen}

Eine wichtige Datenquelle für die Ermittlung des Impfstatus sind die Daten, die im Rahmen der Schuleingangsuntersuchungen jährlich durch die Gesundheitsämter oder von ihnen beauftragten Ärzten nach $₫ 34$ Abs. 11 Infektionsschutzgesetz (IfSG) erhoben werden. Diese aggregierten Daten werden vom Robert Koch-Institut (RKI) zentral erfasst und ausgewertet. Die repräsentativen Impfdaten aus den Schuleingangsuntersuchungen geben 


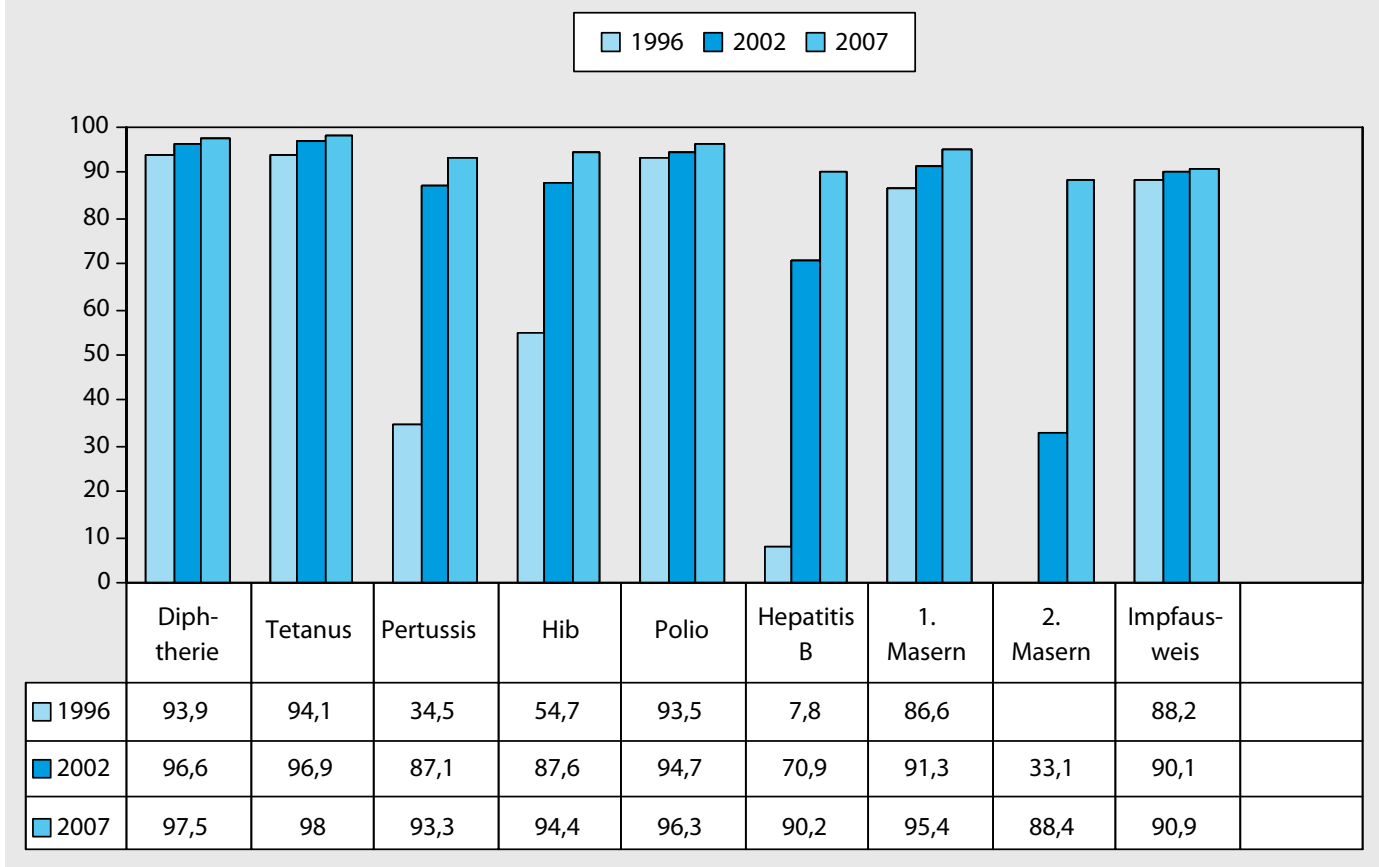

Abb. $1 \varangle$ Impfquote bei den Schuleingangsuntersuchungen in Deutschland 1996/2002/2007

- je nach Einschulungsalter in den Bundesländern - Auskunft über den Impfstatus vier- bis siebenjähriger Kinder. Sie dokumentieren Impfungen, die überwiegend drei bis fünf Jahre zurückliegen, wodurch eine zeitnahe Reaktion auf bestehende Impflücken erschwert wird. Auch liegt bei bis zu $15 \%$ der einzuschulenden Kinder kein Impfausweis vor. Die Schuleingangsuntersuchung selbst ist jedoch auch ein geeigneter, wenngleich sehr später Interventionszeitpunkt, um auf bestehende Impflücken aufmerksam zu machen und sie zu schließen [4].

Die ermittelten Impfquoten werden auf Basis der vorgelegten Impfausweise berechnet, was eine gewisse Überschätzung darstellt. Aus Ausbruchsuntersuchungen und regionalen Untersuchungen ist bekannt, dass die Impfquoten von Kindern ohne Impfausweis in der Regel etwas niedriger sind als bei Kindern mit Impfdokumenten. Im Jahr 2006 wurde bei der Untersuchung eines Masernausbruchs an einer Duisburger Oberschule eine Überschätzung der Impfquote für die erste Masernimpfung durch Bezug auf die vorgelegten Impfausweise um bis zu $8 \%$ festgestellt [5]. Bundesweite Daten liegen jedoch nicht vor.

Die bei den Schuleingangsuntersuchungen dokumentierten Impfquoten lassen über die Jahre einen kontinuierlichen Anstieg erkennen. Ein Vergleich des bei den Schuleingangsuntersuchungen 1996, 2002 und 2007 ermittelten Impfstatus bei
Kindern mit vorgelegtem Impfausweis zeigt, dass die hohen Impfquoten gegen Diphtherie, Tetanus und Poliomyelitis im beobachteten Zeitraum noch leicht angestiegen beziehungsweise auf hohem Niveau konstant geblieben sind ( $\bullet$ Abb. 1).

Besonders deutlich fiel der Anstieg bei den Hib-, Pertussis- Hepatitis-B-, und den zweiten Masernimpfungen aus. Im Jahr 1996 lag die Durchimpfung gegen Pertussis bundesweit bei 34,5\%, 2007 dagegen bei $93,3 \%$. 1996 waren erst 7,8\% der untersuchten Einschüler gegen Hepatitis B geimpft, 2002 waren es $70,9 \%$ und 2007 90,2\%. 2002 betrug die Impfquote für die zweite Masernimpfung 33,1\%, 2007 lag sie bereits bei $88,4 \%$.

Bei den Schuleingangsuntersuchungen 2007 konnten noch nicht alle Bundesländer den neuen zwischen dem RKI und den Bundesländern abgestimmten Meldebogen einsetzen. Dieser sieht eine Erfassung der Varizellen-, Meningokokken-Cund Pneumokokkenimpfungen sowie die Dokumentation der ersten Auffrischimpfungen gegen Tetanus, Diphtherie und Pertussis vor. Um einen bundesweiten Vergleich zu ermöglichen, wurde für die Auswertung der Impfdaten aus den Schuleingangsuntersuchungen 2007 daher nochmals eine Impfserie mit jeweils drei Dosen beziehungsweise vier Dosen für Pertussis als abgeschlossene Grundimmunisierung gewertet. Künftig sind für die Definition eines vollständigen Impfschutzes bei der Verwendung von Impfstoffen mit Pertussiskomponente vier Impfdosen notwendig. Der 2007 ermittelte bundesweite Impfschutz von 705.390 Kindern mit Impfausweis gegen Diphtherie (97\%), Tetanus (98\%), Kinderlähmung (96,3\%) und gegen Hib (94,4\%) ist weiterhin als sehr gut einzustufen (• Tab. 1).

Bei Pertussis sind die bundesweiten Impfquoten weiter leicht angestiegen und liegen mittlerweile in allen Bundesländern deutlich über $90 \%$. Auch für die Hepatitis-B-Impfung ist ein Anstieg auf bundesweit über 90,2\% zu verzeichnen. Für einen ausreichenden Schutz dieser Altersgruppen sind sie aber immer noch unzureichend. Dies gilt auch für den Impfschutz gegen Masern, Mumps und Röteln. Im Vergleich zu 2006 haben sich die bundesweiten Impfquoten für die zweite Masernimpfung von $83,2 \%$ auf $88,4 \%$ erhöht, in den alten Bundesländern von $80,1 \%$ auf $85,8 \%$, in den neuen Bundesländern von $88,5 \%$ auf $91,5 \%$. Dies deutet auf eine zunehmende Akzeptanz und Inanspruchnahme der Masernimpfung hin. Von der für die Elimination notwendigen Impfquote von $95 \%$ für beide Impfungen ist Deutschland jedoch bei der zweite Masernimpfung noch weit entfernt, zumal die ermittelten Impfquoten sich auf Kinder mit Impfausweis beziehen.

Wie auch in den Vorjahren haben die einzuschulenden Kinder in den neuen Bundesländern einen deutlich besse- 
ren Impfschutz gegen Pertussis, Hepatitis B, Masern, Mumps, Röteln als Kinder in den alten Bundesländern. So liegt die Quote für die Hepatitis-B-Impfung in den neuen Bundesländern mit 93,6\% um 3,9\% höher als in den alten Bundesländern. Bei der Grundimmunisierung gegen Pertussis werden in den neuen Bundesländern $96,7 \%$ erreicht, in den alten Bundesländern dagegen $92,9 \%$. Betrachtet man die Spannweite der Impfquoten, die in den Bundesländern durchschnittlich erzielt wurden, so zeigen sich bei der Hib- (91,5\% bis $96,5 \%)$, der Pertussis- (91,3\% bis 97,1\%), der Hepatitis-B-Impfung (80,0\% bis 95,7\%) und bei der zweiten Masernimpfung (8o,9\% bis $94 \%$ ) die größten Unterschiede zwischen den einzelnen Ländern. Der Anteil der vorgelegten Impfausweise reicht von $85,5 \%$ bis $94 \%$.

Die Impfquoten für die seit 2004 empfohlene Varizellenimpfung haben bei den Schuleingangsuntersuchungen 2007 insgesamt neun Bundesländer erfasst. Für die 2007 erfassten Geburtsjahrgänge 2000 bis 2003 spiegeln die Schuleingangsuntersuchungen noch kein realistisches Bild der Akzeptanz der Varizellenimpfung wider. Um die Varizellenimpfquote besser abschätzen zu können, müssen daher noch andere Datenquellen wie die Leistungsund Abrechnungsdaten der Kassenärztlichen Vereinigungen herangezogen werden. Dies gilt auch für die seit $2006 \mathrm{emp}$ fohlenen Impfungen gegen Meningokokken $\mathrm{C}$ und Pneumokokken $[6,7,8]$.

Im internationalen Vergleich für die Europäische Region der Weltgesundheitsorganisation (WHO) erreicht Deutschland mit den Daten aus den Schuleingangsuntersuchungen den europäischen Durchschnitt bei der Diphtherie-, Tetanus- und der Pertussis-Impfung (96\%) sowie bei der ersten Masernimpfung (94\%). Bei der Hepatitis-B-Impfung (78\%) und der Impfung gegen Hib (57\%) liegt Deutschland deutlich über dem europäischen Durchschnitt [9]. Für internationale Vergleiche werden die ermittelten Impfquoten bei Kindern im Alter von 24 Monaten herangezogen. Aktuell liegen diese Daten bisher für Deutschland bundesweit noch nicht kontinuierlich vor; diese Datenlücke soll jedoch durch die Verwendung von Sekundärdaten der Kassenärztlichen Vereinigungen (KVen) geschlossen

Bundesgesundheitsbl 2009 · 52:1037-1044 DOI 10.1007/s00103-009-0957-7

(c) Springer-Verlag 2009

\section{S. Reiter · C. Poethko-Müller \\ Aktuelle Entwicklung von Impfquoten und Impflücken bei Kindern und Jugendlichen in Deutschland}

\section{Zusammenfassung}

Die in Deutschland vorhandenen Datenquellen zum Impfschutz von Kindern und Jugendlichen zeigen einen kontinuierlichen Anstieg der Impfquoten. Der Impfschutz gegen Diphtherie, Tetanus, Polio und Haemophilus influenzae Typ $b$ ist bei Kindern als sehr gut einzustufen. Impfdefizite bestehen bei der Pertussis-, der Hepatitis-B- und den zweiten Masern-, Mumps- und Rötelnimpfungen. Hier sind die für einen effektiven Kollektivschutz und die Masernelimination notwendigen Impfquoten noch nicht erreicht. Die Daten aus dem Kinder- und Jugendgesundheitssurvey (KiGGS) weisen darauf hin, dass Impfungen nicht zeitgerecht, das heißt nicht zu den von der Ständigen Impfkommission (STIKO) empfohlenen Zeitpunkten durchgeführt werden. Insbesondere bei den älteren Kindern und Jugendlichen bestehen noch große Impflücken. Die für diese Altersgrup- pe empfohlenen Auffrischimpfungen gegen Diphtherie, Tetanus, Pertussis und Polio werden nur unzureichend wahrgenommen. Unterschiede bei der Durchimpfung, vor allem in Bezug auf die Vollständigkeit und Zeitgerechtigkeit von Impfungen, gibt es weiterhin zwischen alten und neuen Bundesländern, bei Kindern impfskeptischer Eltern und nicht in Deutschland geborenen Kindern. Eine verbesserte Surveillance des Impf- und Immunstatus und der Impfnebenwirkungen sowie die schnelle Umsetzung eines Nationalen Impfplans könnten die Akzeptanz von Schutzimpfungen erhöhen.

Schlüsselwörter Impfquoten · Impfakzeptanz · Impfprogramme $\cdot$ Kinder und Jugendliche $\cdot$ Datenquellen

\section{Current vaccination coverage and immunization gaps of children and adolescents in Germany}

\section{Abstract}

Available data show increasing vaccination coverage of children and adolescents in recent years in Germany. Vaccination coverage of children against diphtheria, tetanus, polio and haemophilus influenzae Type $b$ is high. Vaccination gaps exist for pertussis, hepatitis $B$ and for the second measles, mumps and rubella doses. Coverage is still insufficient to achieve effective herd immunity and measles elimination. Data from the representative German Health Interview and Examination Survey for Children and Adolescents reveal that vaccination of infants is markedly delayed compared to recommendations of the German Standing Committee on Vaccination (STIKO). Particularly older children and adolescents are often not fully vaccinated or are even unvaccinated. A high pro- portion is missing the recommended booster doses against diphtheria, tetanus, pertussis and polio. Vaccination compliance especially regarding completeness and timeliness is lower in western than eastern Germany, as well as in children of vaccine sceptics and foreign-born children. More comprehensive surveillance of vaccination coverage and adverse events following immunization as well as the implementation of a national immunization plan focussing on closure of immunization gaps could lead to improved vaccination coverage.

\section{Keywords}

Vaccination coverage $\cdot$ Compliance with vaccination $\cdot$ Immunization programme . Children and adolescents · Data sources 
Tab. 1 Durchimpfung und Spannweiten in Prozent der vorgelegten Impfausweise bei den Schuleingangsuntersuchungen in Deutschland 2007 ( $n=705.390$ ). Für Sachsen wurden für Masern, Mumps, Röteln Daten aus den 2. Klassen verwendet, für Diphtherie, Tetanus und Pertussis Daten aus den Kindergartenuntersuchungen

\begin{tabular}{|c|c|c|c|c|c|c|}
\hline \multirow[t]{2}{*}{ Impfung } & \multicolumn{6}{|c|}{ Impfquoten bei Einschülern in \% } \\
\hline & Deutschland & Spannweite ${ }^{\mathrm{a}}$ in \% & $\begin{array}{l}\text { Alte Bundesländer } \\
\text { und Berlin }\end{array}$ & Spannweite $\mathrm{a}^{\mathrm{a}} \%$ & Neue Bundesländer & Spannweite $\mathrm{a}$ in \% \\
\hline Diphtherie & $97,5 \%$ & $94,0-99,1 \%$ & $97,5 \%$ & $94,0-98,5 \%$ & $97,4 \%$ & $96,0-99,1 \%$ \\
\hline Tetanus & $98,0 \%$ & $94,3-99,2 \%$ & $98,1 \%$ & $94,3-98,9 \%$ & $97,7 \%$ & $97,5-99,2 \%$ \\
\hline Pertussis & $93,3 \%$ & $91,3-97,1 \%$ & $92,9 \%$ & $91,3-94,2 \%$ & $96,7 \%$ & $96,2-97,1 \%$ \\
\hline Hib & $94,4 \%$ & $91,5-96,5 \%$ & $94,3 \%$ & $92-95,6 \%$ & $95,1 \%$ & $91,5-96,5 \%$ \\
\hline Poliomyelitis & $96,3 \%$ & $93,9-98,4 \%$ & $96,2 \%$ & $93,9-97,5 \%$ & $96,6 \%$ & $95,0-98,4 \%$ \\
\hline Hepatitis B & $90,2 \%$ & $84,0-95,7 \%$ & $89,7 \%$ & $84,0-93,2 \%$ & $93,6 \%$ & $91,9-95,7 \%$ \\
\hline \multicolumn{7}{|l|}{ Masern } \\
\hline 1. Dosis & $95,4 \%$ & $92,8-98,1 \%$ & $95,0 \%$ & $92,8-96,4 \%$ & $97,6 \%$ & $97,2-98,1 \%$ \\
\hline 2. Dosis & $88,4 \%$ & $80,9-94,0 \%$ & $85,8 \%$ & $80,9-88,1 \%$ & $91,5 \%$ & $87,2-94,0 \%$ \\
\hline \multicolumn{7}{|l|}{ Mumps } \\
\hline 1. Dosis & $95,1 \%$ & $92,5-98,2 \%$ & $94,7 \%$ & $92,5-96,1 \%$ & $97,5 \%$ & $97,0-98,2 \%$ \\
\hline 2. Dosis & $88,1 \%$ & $80,5-93,5 \%$ & $85,5 \%$ & $80,5-87,8 \%$ & $91,3 \%$ & $87,1-93,5 \%$ \\
\hline \multicolumn{7}{|l|}{ Röteln } \\
\hline 1. Dosis & $94,9 \%$ & $92,3-98,1 \%$ & $94,5 \%$ & $92,3-96,0 \%$ & $97,4 \%$ & $96,9-98,1 \%$ \\
\hline 2. Dosis & $87,9 \%$ & $79,3-94,0 \%$ & $85,2 \%$ & $79,3-87,6, \%$ & $91,3 \%$ & $86,8-94,0 \%$ \\
\hline
\end{tabular}

Spannweite über die Bundesländer

werden [2]. In Schleswig-Holstein werden die Abrechnungsziffern der KV zur Dokumentation des Impfstatus von Zweijährigen genutzt. Erste Ergebnisse für die Jahre 2003 bis 2005 zeigen im Vergleich zu anderen früheren Studien für diese Altersgruppe einen Anstieg der Impfquoten wie er im Folgenden auch durch die Daten der KiGGS-Studie beschrieben wird. Die Daten weisen aber auch darauf hin, dass die empfohlenen Impfungen nicht zeitgerecht durchgeführt werden beziehungsweise die Impfserien häufig nicht abgeschlossen werden [10].

\section{Impfquoten und Impflücken bei Kindern aus dem Kinder- und Jugendgesundheitssurvey}

Mit der Durchführung des KiGGS wurden von Mai 2003 bis Mai 2006 insgesamt 17.641 Kinder und Jugendliche im Alter von $0-17$ Jahren untersucht und bei 16.460 Teilnehmern (93,3\%) der Impfstatus auf Grundlage der vorgelegten Impfausweise erfasst; erste Ergebnisse wurden 2007 publiziert [11]. Ein direkter Vergleich der als Querschnittsstudie bei o- bis 17-Jährigen erhobenen Daten der KiGGSUntersuchung mit den Daten der Schuleingangsuntersuchungen einzelner Jahre ist nur eingeschränkt möglich. Ergänzend zu den Daten aus den Schuleingangsun- tersuchungen liefern die KiGGS-Daten Informationen über die Akzeptanz der empfohlenen Boosterimpfungen gegen Diphtherie, Tetanus, Pertussis und Polio. Durch die Erfassung aller im Impfpass dokumentierten Impfdaten kann der Impfstatus zu verschiedenen Alterszeitpunkten zurückgerechnet und damit die Zeitgerechtigkeit von Impfungen beschrieben werden. Darüber hinaus ermöglicht die Erfassung verschiedener soziodemografischer Parameter die Analyse von Determinanten für Impfdefizite. Die KiGGSTeilnehmer/innen von 2003 bis 2006 werden zukünftig in Form einer Kohortenstudie abwechselnd alle drei Jahre telefonisch nachbefragt beziehungsweise nachuntersucht.

\section{Vollständige Grundimmunisierung zum Zeitpunkt des abgeschlossenen zweiten Lebensjahres}

In - Abb. 2 ist die vollständige Grundimmunisierung zum Zeitpunkt des abgeschlossenen zweiten Lebensjahres für Kinder der Geburtsjahrgänge 1987 bis 2003 dargestellt. Dabei wurde für Tetanus, Diphtherie, Hib, Pertussis, Polio und Hepatitis B die Dokumentation von vier Impfdosen als vollständige Grundimmunisierung gewertet. Erfolgte die Impfung mit Impfstoffen ohne Pertussiskomponente, waren drei Impfdosen ausreichend. Während die Quote der Kinder, die am Ende des zweiten Lebensjahres eine unvollständige Grundimmunisierung gegen Tetanus haben, für den Geburtsjahrgang 1987 bei 93,4\% liegt [11], haben nur 57,3\% der Kinder dieses Jahrgangs bis zum zweiten Geburtstag auch die letzte Dosis der Grundimmunisierung (bei Kombinationspräparaten mit Pertussiskomponente also die vierte Impfdosis) erhalten. Für die jüngeren Geburtsjahrgänge 1999 bis 2003 liegt die Impfquote bei über 70\%, ist jedoch deutlich niedriger als die durchgängig über 90\% liegende Quote der unvollständigen Grundimmunisierung. Die Impfquoten der vollständigen Grundimmunisierung gegen Pertussis zum Ende des zweiten Lebensjahres spiegeln die Umsetzung der 1991 ausgesprochenen generellen STIKO-Empfehlung einer Pertussis-Immunisierung wider. Die Impfquoten betrugen bei den Geburtsjahrgängen 1987 bis 1990 zwischen $7,9 \%$ und $15 \%$ (bei hier nicht dargestellten bedeutsamen Ost-WestUnterschieden mit deutlich höheren Impfquoten für Kinder mit Wohnort in den neuen Bundesländern [12]). 1991 begann der kontinuierliche Anstieg der Impfquoten, die bereits 1994 das Niveau der Impfquoten gegen Tetanus erreichten. Auffallend ist, dass - anders als bei den Impfquoten der unvollständigen Grundimmu- 
nisierung - die vollständige Tetanusgrundimmunisierung in den Geburtsjahrgängen 1994 bis 1999 unter der der vollständigen Grundimmunisierung gegen Pertussis lag. Nach der Zulassung der ersten Kombinationspräparate mit azellulärer Pertussiskomponente (ab 1995) scheint die Empfehlung, bei Impfung mit einem Kombinationspräparat mit Pertussiskomponente auch vier Tetanusimpfdosen zu verabreichen, erst zeitversetzt umgesetzt worden zu sein: Viele Kinder erhielten drei Tetanus-Impfdosen mit einem Pertussis-haltigen Kombinationspräparat und anschließend eine vierte Pertussis-Impfung als Monopräparat.

Der Zeitverlauf bei der Umsetzung der neuen STIKO-Empfehlung ist für die Hepatitis-B-Immunisierung ähnlich. Sie wird seit 1995 von der STIKO für Kinder ab dem dritten Lebensmonat empfohlen. Im Unterschied zur Ausgangslage bei der Immunisierung gegen Pertussis lag die Impfquote vor der allgemeinen STIKO-Empfehlung nahezu bei Null und erreichte trotz des zeitnahen Beginns der Empfehlungsumsetzung auch beim Geburtsjahrgang 2001 mit $62,8 \%$ nicht das höhere Niveau der Tetanus-Impfquoten.

\section{Impflücken bei der \\ Pertussis-Impfung}

Insgesamt haben sieben von zehn Kindern im Alter von zwei bis 17 Jahren eine vollständige Grundimmunisierung gegen Pertussis erhalten. Die Impfquoten liegen bei älteren Kindern und Jugendlichen immer noch deutlich unter denen der jeweils jüngeren Altersgruppen. Besonders niedrig sind sie mit 36,1\% bei den 14- bis 17-Jährigen.

In den Geburtsjahrgängen nach der allgemeinen Impfempfehlung gegen Pertussis (1992 bis 2006) sind im Durchschnitt 85,5\% der Kinder im Alter von über einem Jahr vollständig gegen Pertussis grundimmunisiert. Allerdings sind deutliche Defizite bezüglich der zeitgerechten Impfung zu beobachten, was angesichts der besonders hohen gesundheitlichen Gefährdung durch eine Pertussiserkrankung im Säuglingsalter relevant ist. Bereits eine Dosis eines Pertussis-Impfstoffs schützt messbar vor schweren Krankheitsverläufen [13]. Die erste Dosis der Pertussisgrundimmunisierung soll bis zum Ende des

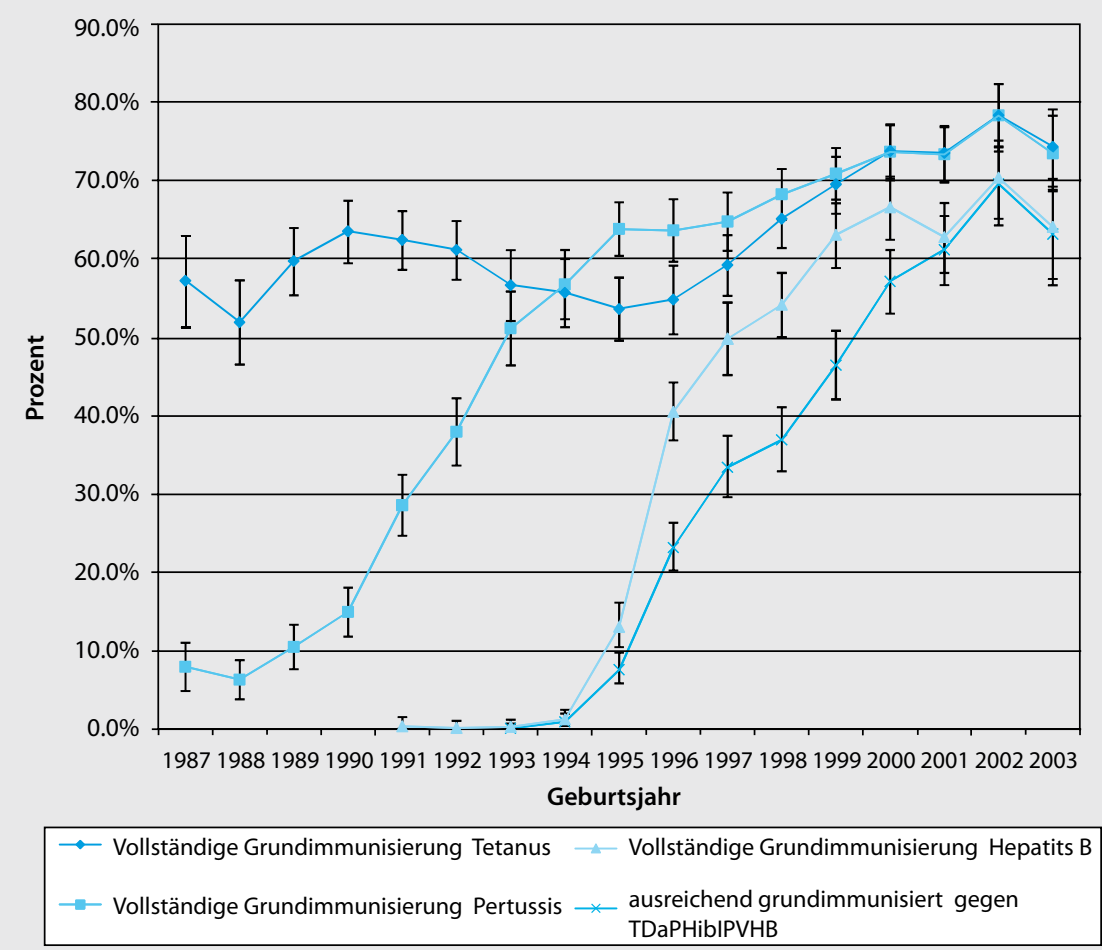

Abb. $2 \Delta$ Impfquoten. Vollständige Grundimmunisierung zum Zeitpunkt des abgeschlossenen zweiten Lebensjahres nach Geburtsjahrgängen; zwei- bis 17-jährige Kinder und Jugendliche. Daten aus dem Kinder- und Jugendgesundheitssurvey (KiGGS)

dritten Monats verabreicht werden. Allerdings erhielten im Durchschnitt nur 20,4\% der Kinder eine erste Impfdosis bis zum Ende des dritten Lebensmonats. Der Anteil der Kinder mit zeitgerechter erster Pertussis-Impfung hat sich jedoch in den Jahren 1992 bis 2006 kontinuierlich erhöht: Während in den Jahrgängen 1992 bis 1993 nur 7,3\% der Kinder die erste Impfung zeitgerecht erhalten hatten, waren es in den Jahrgängen 2002 bis 2006 bereits $32,6 \%$. Auch hinsichtlich der Vollständigkeit der Grundimmunisierung zum Ende des zweiten Lebensjahres ist in den vergangenen Jahren eine positive Entwicklung zu beobachten: Während in den Geburtsjahrgängen 1992 bis 1993 nur 44,4\% Kinder zum zweiten Geburtstag vollständig gegen Pertussis grundimmunisiert waren, waren es in den Jahrgängen 2002 bis 2006 bereits $76,6 \%$ aller Kinder. Allerdings bedeutet das noch immer, das die Vollständigkeit der Grundimmunisierung für zu viele Kinder erst spät gegeben ist: Fast jedes vierte Kind hatte zum zweiten Geburtstag noch keine ausreichende Grundimmunisierung gegen Pertussis.

Der Anteil von Kindern mit zeitgerechter erster Pertussis-Impfung unterschied sich sehr deutlich zwischen den alten und neuen Bundesländern: Während in den neuen Bundesländern 37,5\% der Kinder ihre erste Impfung zeitgerecht erhielten, waren es in den alten Bundesländern nur 17,3\%

(- Abb. 3). Ost-West-Unterschiede bestehen auch hinsichtlich der vollständigen Keuchhustengrundimmunisierung zum Ende des zweiten Lebensjahres, der Unterschied ist jedoch weniger deutlich als der in der Zeitgerechtigkeit der ersten Impfung.

\section{Impflücken bei der Masernimpfung}

Die erste Masernimpfung haben den KiGGS-Daten zufolge 93,6\% aller Kinder im Alter von zwei bis 17 Jahren erhalten. Bei drei Vierteln aller Kinder wurde auch eine zweite Masernimpfung durchgeführt. Die STIKO-Empfehlung einer zweiten Maserndosis besteht seit 1991. Diese wurde zunächst für das sechste bis siebte Lebensjahr empfohlen, seit 2001 soll die zweite Impfdosis (wie auch für Mumps und Röteln) bereits im zweiten Lebensjahr gegeben werden. Dass sich diese Empfehlung noch nicht ausreichend durchgesetzt hat, zeigt die in KiGGS festgestellte niedrige Impfquote für die zweite Masernimpfung bei den Zweijährigen. Dies bestätigt die in Schleswig-Holstein erhobenen Daten [3]. Jedoch weisen 


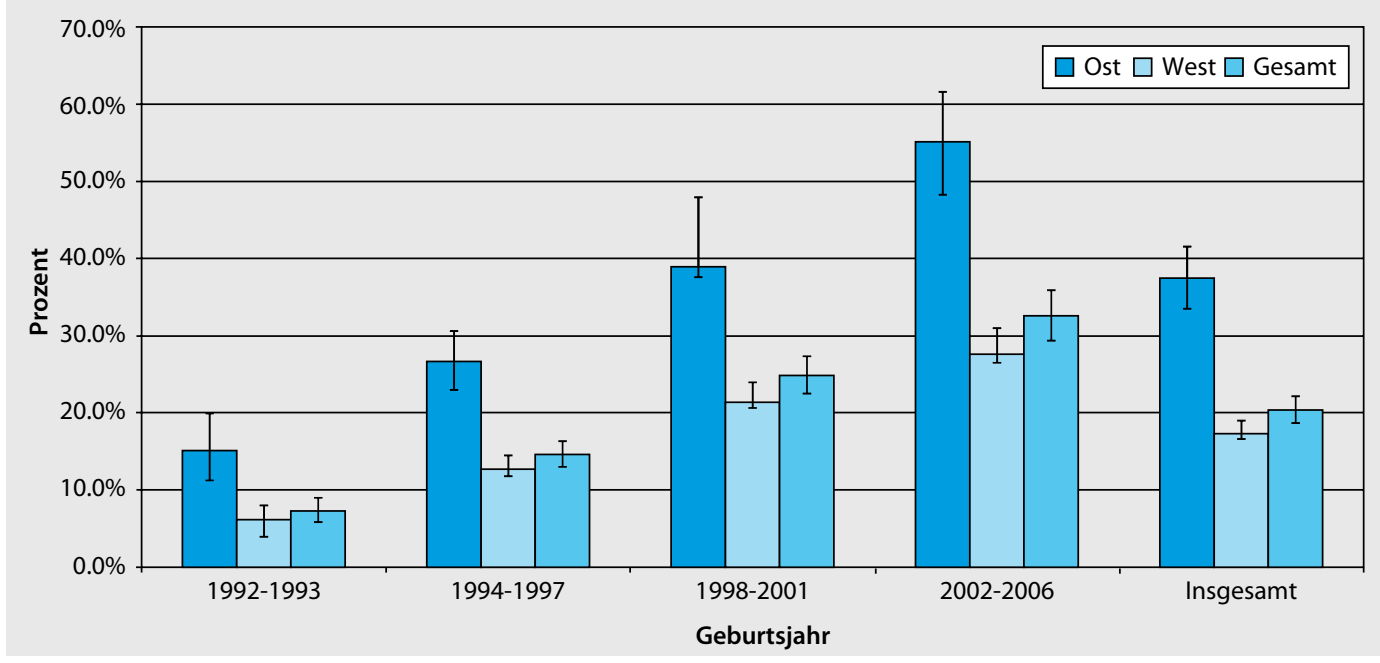

Abb. $3<$ Anteil der Kinder mit erster Keuchhustenimpfung bis zum 90. Lebenstag. Kinder der Geburtsjahrgänge 19922006. Daten aus dem Kinder- und Jugendgesundheitssurvey (KiGGS)

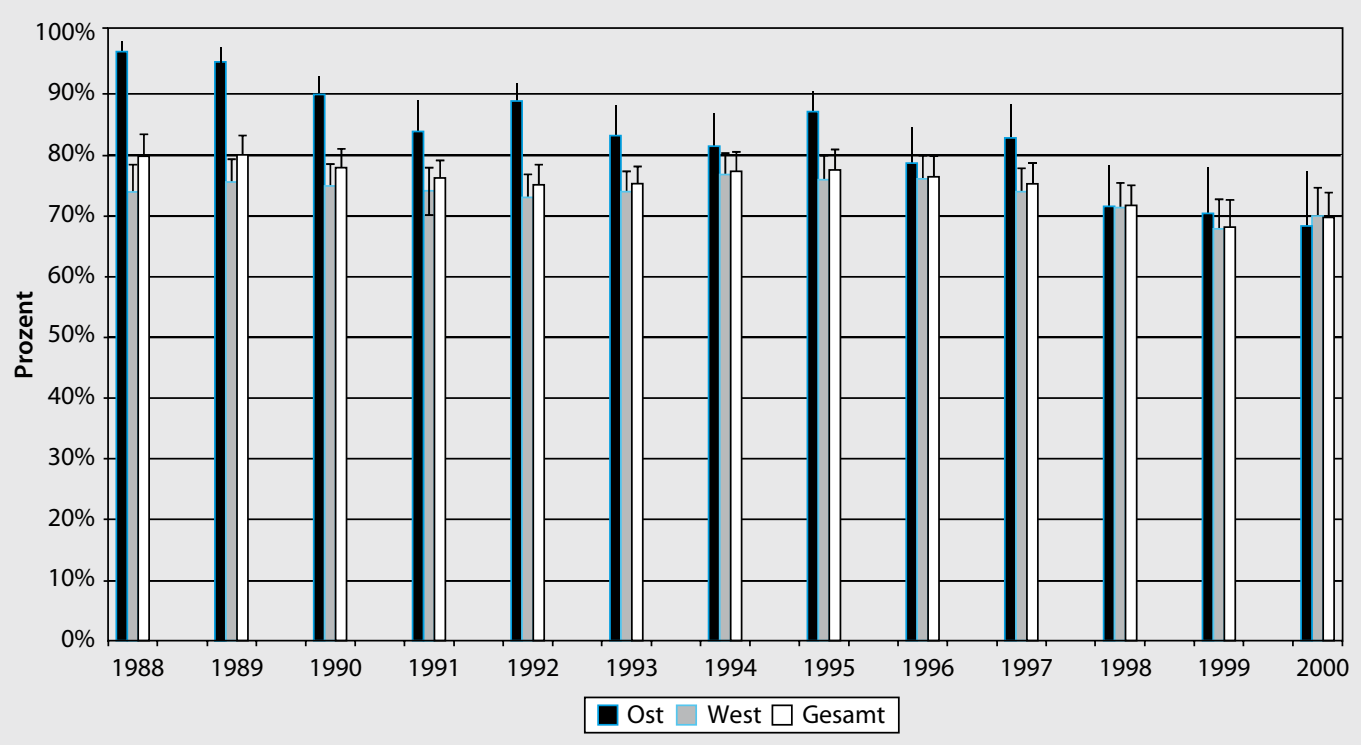

Abb. $4<$ Impfquoten. Vollständige Masernimpfung (zwei Impfungen) nach Geburtsjahrgang; zwei- bis 17-jährige Kinder und Jugendliche. Daten aus dem Kinder- und Jugendgesundheitssurvey (KiGGS) auch ältere Kinder und Jugendliche deutliche Defizite auf. Insbesondere bei den älteren Kindern und Jugendlichen bestehen noch sehr große Unterschiede zwischen den alten und neuen Bundesländern. Während in den Jahrgängen vor 1990 die Durchimpfung mit zwei Masernimpfungen in den neuen Bundesländern bei über 90\% liegt (• Abb.4), liegt sie in den alten Bundesländern bei wenig über $70 \%$.

Deskriptive und multivariate Auswertungen zeigen, dass besonders bei Kindern mit mehr als drei Geschwistern, bei selbst zugewanderten Migranten, bei Kindern aus den alten Bundesländern und Kindern, deren Eltern Vorbehalte gegen Impfungen äußern und das Durchmachen von Erkrankungen für besser halten als die Impfung, der Anteil ungeimpfter Kinder (und von Kindern, denen die zweite Impfung fehlt) groß ist [14]. Von den Kin- dern im Alter von zwei bis 17 Jahren, deren Eltern die genannten Vorbehalte gegen Impfungen haben, sind 54\% nicht gegen Masern geimpft.

\section{Impflücken bei der \\ Hepatitis-B-Impfung}

Eine vollständige Grundimmunisierung gegen Hepatitis B haben zwei Drittel aller Kinder im Alter von zwei bis 17 Jahren erhalten. Die Impfquoten sind bei den Elf- bis 17-Jährigen deutlich niedriger als bei den Zwei- bis Zehnjährigen. Die meisten Kinder und Jugendlichen der höheren Altersgruppen sind in den Jahren 1985 bis 1994 also vor der 1995 durch die STIKO ausgesprochenen allgemeinen Empfehlung der Hepatitis-B-Impfung - geboren und wurden bislang nicht in ausreichendem Umfang nachgeimpft. Mit 58,3\% ist die Impf- quote für die vollständige Grundimmunisierung in der Altersgruppe der 14- bis 17Jährigen besonders niedrig und liegt deutlich unter der für alle Kinder und Jugendlichen erwünschten Durchimpfung gegen Hepatitis B von über 95\%.

\section{Impflücken bei Auffrischungsimpfungen}

Nur 57,0\% aller Kinder im Alter von sieben und zehn Jahren haben die für das sechste bis siebte Lebensjahr empfohlene Auffrischimpfung gegen Tetanus erhalten. Die Impfquoten dieser ersten Auffrischimpfung gegen Tetanus liegen bei älteren Kindern und Jugendlichen höher und erreichen bei den 14- bis 17-Jährigen $88,1 \%$. 53,8\% der Elf- bis 17 -Jährigen haben zusätzlich zur Poliogrundimmunisierung eine Auffrischungsimpfung erhalten 
(- Abb. 5). Der Anteil der Kinder, bei denen zusätzlich zur abgeschlossenen Grundimmunisierung eine fünfte PertussisImpfung dokumentiert war (Grundimmunisierung mit erster Auffrischung) ist in der Altersgruppe der Elf- bis 13-Jährigen 13,6\%. Wie auch bei der Tetanusauffrischung bestehen deutliche Ost-West-Unterschiede (Ost: 32,5\%; West: 10,5\%). Bei der Interpretation der Ergebnisse muss jedoch bedacht werden, dass die erste Auffrischimpfung gegen Pertussis während der Studienlaufzeit noch für das Alter neun bis 17 Jahre empfohlen war [12]. Eine aktuelle Arbeit [15] hat gezeigt, dass die einmalige Impfung mit einem TdaP-Impfstoff bei über zehnjährigen Ungeimpften zu ähnlichen Antikörper-Antworten führt wie bei Personen mit erfolgter Grundimmunisierung. Daher wurden zusätzlich zu den Kindern mit vollständiger Pertussis-Grundimmunisierung einschließlich erfolgter Auffrischungsimpfung auch die Kinder betrachtet, die im Alter von über zehn Jahren mindestens eine Pertussis-Impfung erhalten haben. Es zeigt sich jedoch, dass dennoch fast $80 \%$ der Elfbis 17-Jährigen keinen ausreichenden Impfschutz gegen Pertussis haben.

\section{Schlussfolgerungen und Ausblick}

Die in Deutschland vorhandenen Datenquellen zum Impfschutz von Kindern und Jugendlichen zeigen einen kontinuierlichen Anstieg der Impfquoten. Der Impfschutz gegen Diphtherie, Tetanus, Polio und Haemophilus influenzae Typ b ist für die Altersgruppe der Kinder weiterhin als sehr gut einzustufen. Defizite bestehen vor allem beim Impfschutz gegen Pertussis, Hepatitis B und den zweiten Impfungen gegen Masern, Mumps und Röteln. Hier müssen die Impfquoten für alle Altersgruppen erhöht und Impfungen nachgeholt werden. Zur Akzeptanz der 2004 und 2006 empfohlenen Impfungen gegen Varizellen, Pneumokokken und Meningokokken liegen noch keine aktuellen bundesweiten Daten vor, ebenso wenig wie zur HPV-Impfung.

Die bei Kindern im Alter von 24 Monaten erzielten Impfquoten gelten weltweit als epidemiologisch sinnvoller und wichtiger Indikator für die Evaluation nationaler Impfprogramme beziehungsweise Impfempfehlungen [16]. Die KiGGS-

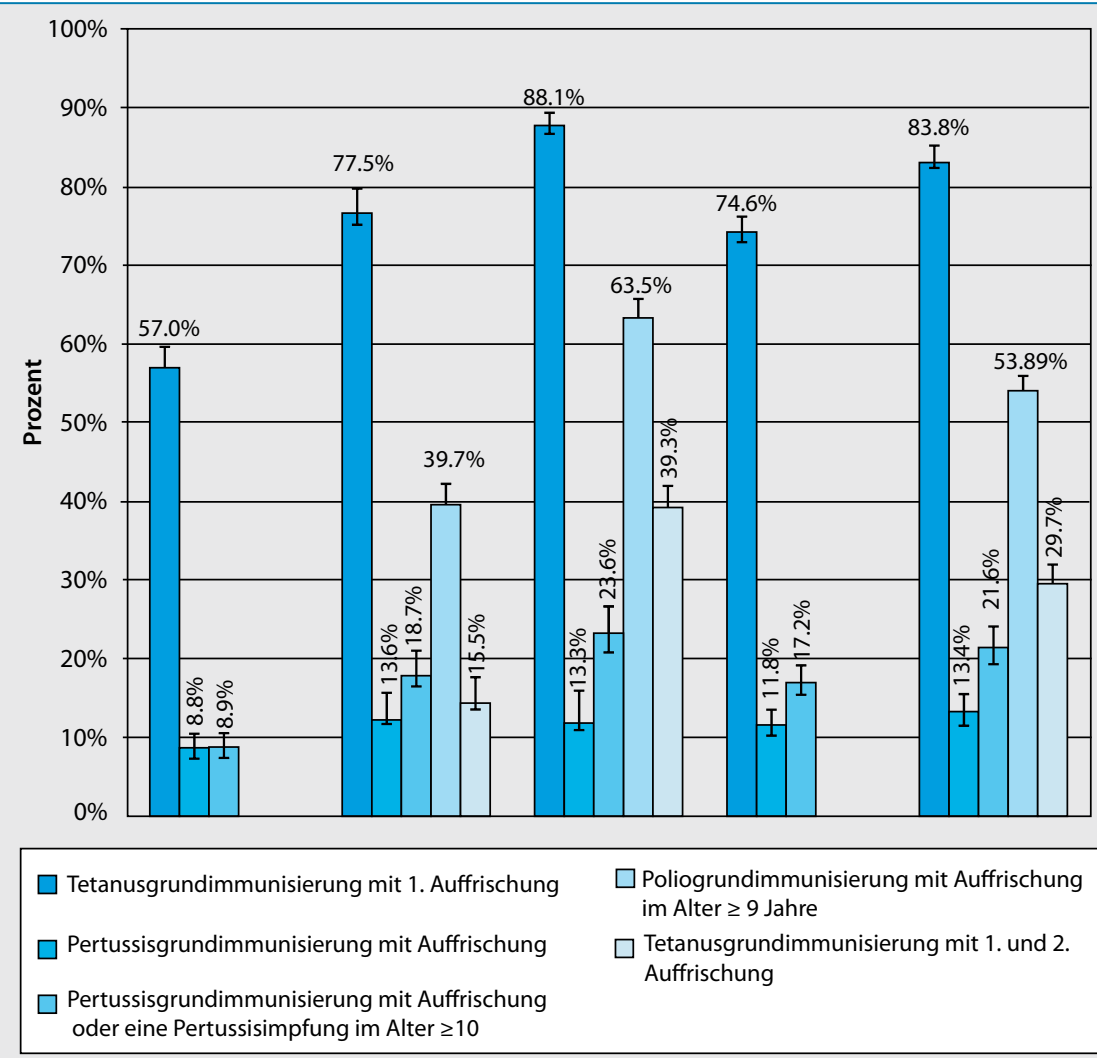

Abb. $5 \Delta$ Impfquoten für Auffrischungsimpfungen nach Alter, sieben- bis 17-jährige Kinder und Jugendliche. Daten aus dem Kinder- und Jugendgesundheitssurvey (KiGGS)

Daten und Ergebnisse früherer Untersuchungen [10] belegen zum einen den gestiegenen Anteil der Kinder mit vollständiger Grundimmunisierung am Ende des zweiten Lebensjahrs über die Zeit. Zum anderen verdeutlichen sie, dass die Impfquoten zu diesem Lebensalter deutlich zu niedrig sind.

Um das für die europäische Region der WHO angestrebte Ziel der Masernelimination bis 2010 erreichen zu können, müssen bundesweit noch verstärkte Anstrengungen bei der Erhöhung der Impfakzeptanz und einem effektiven Fallmanagement bei Auftreten von Masernfällen unternommen werden. Regionale Ausbrüche von Maserninfektionen - 2006 in Nordrhein-Westfalen [17, 18] und Niederbayern [19], 2007/2008 in der Schweiz mit Ausbreitung nach BadenWürttemberg [20], die Masernausbrüche 2008 und 2009 [21] - zeigen die bislang noch unbefriedigende Situation beim Stand der Masernelimination in Deutschland. Besonders der Masernausbruch in Nordrhein-Westfalen 2006 mit 1749 Fällen, von denen $15 \%$ hospitalisiert werden mussten und zwei verstarben, verdeutlicht die Notwendigkeit gezielter Impf- programme und der Impflückenschließung vor allem im Jugendalter. Die meisten Masernfälle waren dort in der Altersgruppe der Zehn- bis 14-Jährigen aufgetreten [22].

Die KiGGS-Daten und die vorliegenden Daten aus einigen Bundesländern zum Impfschutz Jugendlicher machen deutlich, dass in dieser Altersgruppe große Impflücken bestehen. Sie zeigen, dass die empfohlenen Boosterimpfungen für Jugendliche nur unzureichend durchgeführt und fehlende Impfungen nicht genügend nachgeholt werden. Es gibt nur wenige Bundesländer, in denen der Öffentliche Gesundheitsdienst (ÖGD) den Impfstatus in dieser Altersgruppe regelmäßig und flächendeckend überprüft [23]. Kontinuierliche Impfstatusüberprüfungen und Impflückenschließungen sind für diese Altersgruppe notwendig ebenso wie ein Erfassungssystem für die durchgeführten HPV-Impfungen Für die Altersgruppe der Jugendlichen spielt der ÖGD neben den niedergelassenen Ärzten eine wichtige Rolle, da die Jugendgesundheitsuntersuchung für die Zwölf- bis 14-Jährigen nur von einem Drittel der Jugendlichen in Anspruch ge- 
nommen wird. Zahlreiche Impfprojekte belegen, dass Jugendliche über Schulen gut zu erreichen sind. Am erfolgreichsten haben sich dabei Aktionen des ÖGD erwiesen, die neben der Kontrolle der Impfausweise auch Impfungen anbieten und damit auch eine sozialkompensatorische Aufgabe erfüllen [23].

Unterschiede hinsichtlich Vollständigkeit und Zeitgerechtigkeit der empfohlenen Impfungen zeigten sich auch zwischen Kindern aus den alten und neuen Bundesländern, die Vollständigkeit differiert zwischen den Alters- und Sozialstatusgruppen und Kindern mit beziehungsweise ohne Migrationshintergrund.

Die Ursachen für die teilweise unzureichende Impfakzeptanz und die bestehenden Impflücken sind vielfältig und komplex. Sie sind durch strukturelle und organisatorische Faktoren im Gesundheitswesen, aber auch durch soziale, historische, kulturelle und weltanschauliche Faktoren beeinflusst. Zu den häufigsten Gründen gehören ein unzureichendes Wissen über den Wert und die Notwendigkeit von Impfungen, die Angst vor Nebenwirkungen, falsche Kontraindikationen, die Verunsicherung durch Impfgegner und Impfskeptiker, eine bewusste Entscheidung gegen das Impfen, vor allem aber die unzureichende Nutzung des Arzt-Patienten-Kontaktes und Vergessen durch fehlende Erinnerungssysteme [14, 24, 25].

Um die Impfquoten bei Kindern und Jugendlichen und in der Gesamtbevölkerung zu erhöhen, die Barrieren bei Impfungen für sozial benachteiligte Gruppen zu verringern und auch Impfskeptiker zu überzeugen, müssen alle Akteure in der Impfprävention enger zusammenarbeiten. Die Etablierung von Recall-Systemen und die systematische Nutzung der Vorsorgeuntersuchungen für Impfungen, die gezielte Ansprache von Migranten und von Eltern mit Vorbehalten gegen Impfungen sind weitere notwendige Maßnahmen zur Erhöhung der Impfakzeptanz. In Deutschland gibt es öffentliche Impfempfehlungen, jedoch kein nationales Impfprogramm. Es fehlen teilweise auch Strukturen, um die Umsetzung von Impfzielen koordiniert verfolgen und den Impferfolg zeitnah und valide messen zu können.
Für die USA und Israel konnte gezeigt werden, dass eine Erhöhung der Impfquoten durch verbesserte kleinräumige Impferfassungssysteme wie Impfregister möglich ist [26]. Für Deutschland werden bundesweite aktuelle Daten zum zeitgerechten Impfschutz von Kleinkindern benötigt, damit frühzeitig Impflücken geschlossen werden können. Erforderlich sind aber auch kontinuierlich erhobene repräsentative, aktuelle Daten zum Impfschutz von Kindern, Jugendlichen, Erwachsenen sowie von Risikogruppen. Die 2008 publizierte Strategie der Bundesregierung zur Förderung der Kindergesundheit, deren Ausgangspunkt die Ergebnisse des KiGGS sind, zielt auf eine Steigerung der Impfquoten bei Masern, Röteln, Keuchhusten und Hepatitis B auf 95\% durch Impfkampagnen und zielgruppenspezifische Öffentlichkeitsarbeit. Ziel ist es zudem, die bereits erreichten Impfquoten von über $95 \%$ bei Poliomyelitis, Diphtherie und Tetanus aufrechtzuerhalten [27]. Auf der 1 Nationalen Impfkonferenz, die vom 5.-7. März 2009 in Mainz stattfand, wurden erste Ziele und Prioritäten als Empfehlungen für einen „Nationalen Impfplan“ formuliert. Die Umsetzung eines solchen Plans wäre ein weiterer wichtiger Schritt zu einem verbesserten Impfschutz der Bevölkerung.

\section{Korrespondenzadresse}

\section{Dr. S. Reiter}

Abteilung für Infektionsepidemiologie,

Fachgebiet Impfprävention,

Robert Koch-Institut

13302 Berlin

ReiterS@rki.de

Interessenkonflikt. Der korrespondierende Autor gibt an, dass kein Interessenkonflikt besteht.

\section{Literatur}

1. RKI (2008) Empfehlungen der Ständigen Impfkommission (STIKO) am Robert Koch-Institut. Epid Bull 30:235-254

2. Poggensee G, Reuss A, Reiter S, Siedler A (2009) Überblick und Bewertung der verfügbaren Datenquellen zur Inzidenz impfpräventabler Krankheiten, zum Durchimpfungsgrad und zum Immunstatus. Bundesgesundheitsbl Gesundheitsforsch Gesundheitsschutz 52

3. Bader HM, Rasche S (2009) Impfschutz bei Aufnahme in den Kindergarten 2007. Schleswig-Holsteinisches Ärztebl 3:57-61

4. Sing A, Wildner M, Zapf A (2008) Impf-Recall bei Schuleingangsuntersuchungen führt zur Steigerung der Impfquoten. Ergebnisse eines Pilotprojekts am Gesundheitsamt Pfaffenhofen. Epid Bull 16:123-125

5. Wichmann O, Hellenbrand W, SagebiehI D et al (2007) Large measles outbreak at a German public school, 2006. Pediatr Infect Dis J 26:782-786
6. Reuss A, Poggensee G, Eckmanns T (2008) Zum Vergleich der Häufigkeit von Varizellenimpfungen anhand von Erhebungen im Rahmen des KV-Sentinels des RKI und des Varizellen-Sentinels der AGMV. Epid Bull 8:64-

7. Siedler A (2008) Zur Sentinel-Surveillance der Varizellen in Deutschland. Epid Bull 8:61-64

8. Kalies H, Redel R, Varga R et al (2008) Vaccination coverage in children can be estimated from health insurance data. BMC Public Health 8:82

9. WHO (2209) WHO vaccine-preventable disease monitoring system, 2008 global. http://www.who.int/immunization_monitoring/en/globalsummary/GS_EURProfile.pdf

10. Bader H, Ludwig M (2007) Zu den Impfungen bei Kindern im Alter bis zu 24 Monaten. Eine Erhebung in Schleswig-Holstein nach Abrechnungsziffern der Kassenärztlichen Vereinigung für 2003 bis 2005. Epid Bull 34:316-321

11. Poethko-Müller C, Kuhnert R, Schlaud M (2007) Durchimpfung und Determinanten des Impfstatus in Deutschland - Ergebnisse des Kinder- und Jugendgesundheitssurveys (KiGGS). Bundesgesundheitsbl Gesundheitsforsch Gesundheitsschutz 50:851-862

12. Poethko-Müller C (2008) Pertussis: Zum Vergleich von Grundimmunisierung und Auffrischimpfungen in den alten und neuen Bundesländern. Daten aus dem Kinder- und Jugendgesundheitssurvey (KiGGS). Epid Bull 27:213-215

13. Riffelmann M, Littmann M, Hellenbrand W et al (2008) Pertussis - nicht nur eine Kinderkrankheit. Dtsch Ärztebl 105:623-628

14. Poethko-Müller C, Ellert U, Kuhnert R et al (2009) Vaccination coverage against measles in German-born and foreign-born children and identification of unvaccinated subgroups in Germany. Vaccine 27:2563-2569

15. Knuf M, Zepp F, Meyer C et al (2006) Immunogenicity of a single dose of reduced-antigen acellular pertussis vaccine in a non-vaccinated adolescent population. Vaccine 24(12):2043-2048

16. Reiter S (2004) Ausgewählte Daten zum Impf- und Immunstatus in Deutschland. Bundesgesundheitsbl Gesundheitsforsch Gesundheitsschutz 47:1144-1150

17. Horacek U (2007) Masern: Zu einem Ausbruch in NRW 2006 - Konsensuspapier einer Arbeitsgruppe der beteiligten Gesundheitsämter. Epid Bull 13:109-112

18. Santibanez S (2007) Masern - Zur Situation in Deutschland. Epid Bull 37:341-344

19. Bernard H, Siedler A (2007) Zu einem Masernausbruch in Niederbayern im 1. Halbjahr 2007. Epid Bull 37:344347

20. Pfaff G, Mezger B, Santibanez S, Hofmann U et al (2008) Masles in southwest-Germany imported from Switzerland - a preliminary outbreak description. Euro Surveillance 13(8):pii=8044

21. Richard J, Masserey-Spicher V, Santibanez S, Mankertz A (2008) Measles outbreak in Switzerland - an update relevant for the European Football Championship (EURO 2008). Euro Surveillance 13(8):pii8043

22. Wichmann O, Siedler A, Sagebiehl D et al (2009) Further efforts needed to achieve measles elimination in Germany: results of an outbreak investigation. Bull World Organ 87:108-115

23. Elsäßer G (2008) Jugendimpfung - Herausforderung und Chancen. Kinderärztliche Praxis 79:31-35

24. Schmitt HJ (2002) Factors influencing vaccine uptake in Germany. Vaccine 20:S2-S4

25. Meyer C, Reiter S (2004) Impfgegner und Impfskeptiker. Bundesgesundheitsbl Gesundheitsforsch Gesundheitsschutz 47:1144-1150

26. Papania M, Rodewald L (2006) For better immunisation coverage, measure coverage better. Lancet 367:965-966

27. Bundesministerium für Gesundheit (2008) Strategie der Bundesregierung zur Förderung der Kindergesundheit. Berlin http://www.bmg.bund.de/cln_153/ SharedDocs/Publikationen/DE/Praevention/StrategieKindergesundheit,templateld=raw,property=publicationFile.pdf/Strategie-Kindergesundheit.pdf 